High Energy Phenomena in Relativistic Outflows (HEPRO IV)

International Journal of Modern Physics: Conference Series

Vol. 28 (2014) 1460176 (7 pages)

(C) The Authors

DOI: 10.1142/S2010194514601768

\title{
THE FSRQs 3C 279 AND PKS 1510-089: MAGIC LATEST RESULTS AND MULTIWAVELENGTH OBSERVATIONS*
}

\author{
G. DE CANEVA ${ }^{1, \dagger}$, U. BARRES DE ALMEIDA ${ }^{2}$, E. LINDFORS ${ }^{3}$, K. SAITO ${ }^{4}$, \\ C. SCHULTZ ${ }^{5}$, J. SITAREK 6 and F. TAVECCHIO ${ }^{7}$ \\ (for the MAGIC Collaboration),

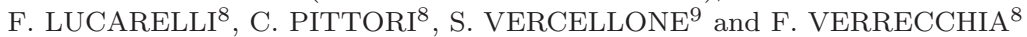 \\ (for the AGILE Collaboration), \\ S. BUSON ${ }^{5}$, F. D'AMMANDO ${ }^{10}$ and M. HAYASHIDA ${ }^{11}$ \\ (for the Fermi-LAT Collaboration), \\ A. LAHTEENMAKI ${ }^{12}$, M. TORNIKOSKI ${ }^{12}$, T. HOVATTA ${ }^{13}$, C. MUNDELL ${ }^{14}$, \\ I. STEELE ${ }^{14}$, K. NILSSON ${ }^{3}$, A. MARSCHER ${ }^{15}$ and S. JORSTAD ${ }^{15}$ \\ ${ }^{1}$ Deutsches Elektronen-Synchrotron (DESY), D-15738 Zeuthen, Germany \\ ${ }^{2}$ Max-Planck-Institut für Physik, D-80805 München, Germany \\ ${ }^{3}$ Finnish Centre for Astronomy with ESO (FINCA), University of Turku, Finland \\ ${ }^{4}$ Japanese MAGIC Consortium, Division of Physics and Astronomy, \\ Kyoto University, Japan \\ ${ }^{5}$ Università di Padova and INFN, I-35131 Padova, Italy \\ ${ }^{6}$ IFAE, Edifici Cn., Campus UAB, E-08193 Bellaterra, Spain \\ ${ }^{7}$ INAF National Institute for Astrophysics, I-00136 Rome, Italy \\ ${ }^{8}$ ASI Science Data Center (ASDC), via del Politecnico snc, \\ 00133 Roma and INAF-OAR, Via Frascati 33, \\ I00040 Monte Porzio Catone (RM), Italy \\ ${ }^{9}$ INAF-IASF Palermo, Via Ugo La Malfa 153, 90146 Palermo, Italy \\ ${ }^{10}$ INAF-IRA, I-40129 Bologna, Italy \\ ${ }^{11}$ Institute Cosmic-ray research, University of Tokyo, \\ Kashiwa, 277-8582, Chiba, Japan \\ 12 Aalto University Metsähovi Radio Observatory, \\ Metsähovintie 114, 02540, Kylmälä, Finland \\ ${ }^{13}$ Cahill Center for Astronomy \& Astrophysics, Caltech, \\ 1200 E. California Blvd, Pasadena, CA, 91125, USA \\ ${ }^{14}$ Astrophysics Research Institute, Liverpool John Moores University, \\ Twelve Quays House, Egerton Wharf, Birkenhead, CH41 1LD, UK \\ ${ }^{15}$ iNSTITUTE for Astrophysical Research, Boston University, USA \\ ${ }^{\dagger}$ gessica.de.caneva@desy.de
}

Received 31 October 2013

Revised 26 November 2013

Published 21 March 2014

At very high energy (VHE, E $>100 \mathrm{GeV}$ ), we count only three blazars of the flat spectrum radio quasars (FSRQs) type to date. The MAGIC experiment detected all three of them;

\footnotetext{
* This is an Open Access article published by World Scientific Publishing Company. It is distributed under the terms of the Creative Commons Attribution 3.0 (CC-BY) License. Further distribution of this work is permitted, provided the original work is properly cited.
} 
here we present MAGIC observations of 3C 279 and PKS 1510-089. 3C 279 was observed in 2011, without a significant detection, hence upper limits on the differential flux have been computed. The MAGIC observations of PKS 1510-089 in 2012 were triggered by alerts of high activity states and resulted in a significant detection. MAGIC observations are complemented with simultaneous multiwavelength observations in high energy $\gamma$ rays, $\mathrm{X}$-rays, optical and radio wavelengths and polarization measurements. With the study of the spectral features and the variability observed, we aim to identify the physical processes responsible for the behavior of this source class. In particular, we propose coherent scenarios, which take into account both the modeling of the spectral energy distribution and the constraints obtained from the lightcurves.

Keywords: Quasars: individual (3C 279, PKS 1510-089); gamma rays; active galaxies; radiation mechanism: non-thermal.

PACS numbers: 95.85.Pw, 98.54.Aj, 95.30.Gv

\section{Introduction}

FSRQs are a subclass of blazars characterized by pronounced emission lines in the optical spectrum. Out of the 52 blazars $^{\text {a }}$ known to be VHE $\gamma$-ray emitters, only three are FSRQs: $3 \mathrm{C} 279,{ }^{1}$ PKS $1510-089^{2,3}$ and PKS $1222+21 .^{4}$ The MAGIC experiment, stereo Cherenkov telescopes sensitive to $\mathrm{VHE} \gamma$ rays located in the Canaries, ${ }^{5}$ detected all three of them. In this work we are presenting MAGIC results together with multiwavelength (MWL) observations for the sources 3C $279^{10}$ and PKS 1510-089. ${ }^{11}$

\section{3 C 279}

3C 279 is the first FSRQ detected at $\mathrm{VHE}^{1}$ and among the most distant known sources at VHE. In 2011 it was observed with the MAGIC telescopes twice as part of two different campaigns: ${ }^{10}$ from February to April for regular monitoring and, in June, after alerts of high activity states in optical and high energy $\gamma$ rays (HE, $100 \mathrm{MeV}<\mathrm{E}<100 \mathrm{GeV}$ ). No signal was found in any of the observation periods, hence upper limits on the differential flux have been derived (left panel of Fig. 1). We have complemented VHE data with MWL observations at lower energies (right panel of Fig. 1 and Fig. 2). During spring 2011 (Fig. 2) there were two subsequent flares in the HE band, with the same trend in the X-ray band and an optical flare started during the descending phase of the second HE flare. Simultaneously with the HE $\gamma$ rays and optical flares, there was a rotation of the optical polarization angle, as observed in previous flares. ${ }^{6}$ Contrary to previous flaring episodes of this source, no enhanced activity has been measured in the radio band and new radio knots were not ejected. The behavior observed in June 2011 (right panel of Fig. 1) can be interpreted with a two-zone leptonic model. ${ }^{8} \mathrm{X}$-ray, HE and VHE emission is generated in a region inside the broad line region (long-dashed line), while the optical emission comes from an external region, located close to the radio core (short-dashed line). The

\footnotetext{
a http://tevcat.uchicago.edu/
} 

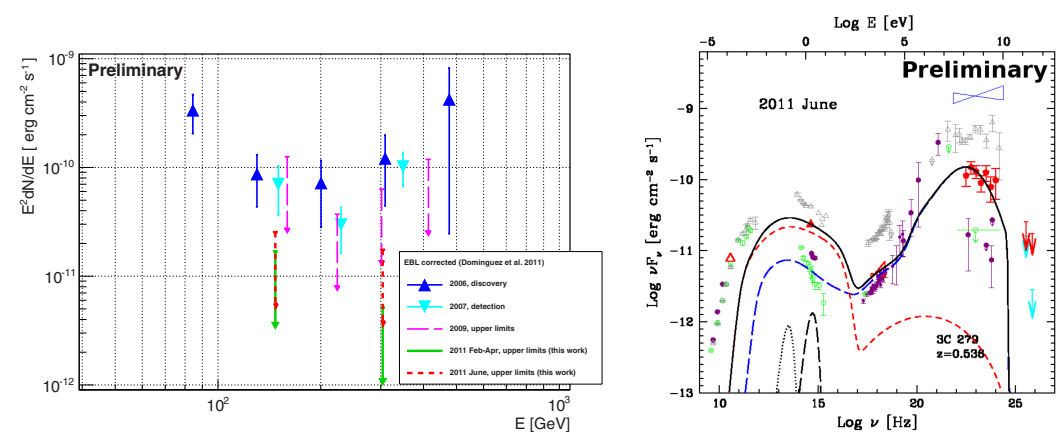

Fig. 1. (Left) MAGIC observations of 3C 279 (see Ref. 10): upper limits obtained from 2011 observations and historical observations (Ref. 6). All observations are corrected for the absorption of the extragalactic background light (EBL) using the model of Ref. 7. (Right) The MWL SED obtained for June observations of 3C 279: radio from Metsähovi (red open triangle), optical from KVA (red full triangles), X-ray from RXTE (red bowtie), HE $\gamma$ rays from Fermi (red circles) and MAGIC (red arrows: EBL corrected, cyan arrows: observed points). Historical data are also shown (Ref. 6).

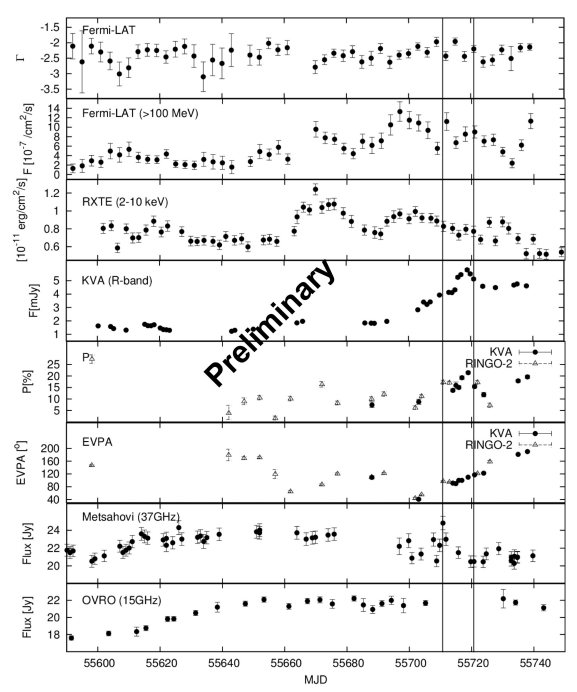

Fig. 2. The MWL lightcurves of 3C 279 from February to June 2011 (see Ref. 10).

features of the rotation in polarization angle have been interpreted with a bent trajectory of the emission feature, amplified due to relativistic aberration.

\section{PKS 1510-089}

In early 2012, PKS 1510-089 was flaring in the HE $\gamma$-ray band; after alerts from the AGILE and Fermi-LAT collaborations, MAGIC performed follow-up observations. The source was detected at $\mathrm{VHE}^{3}$ and found that the spectra is very soft (the observed spectral index is $4.0 \pm 0.4$, while the index of the EBL corrected flux is 


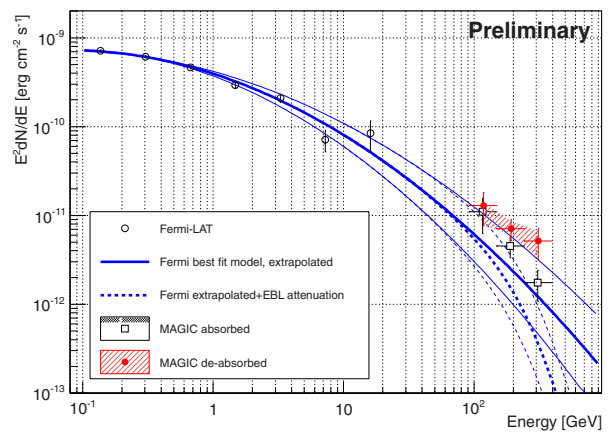

Fig. 3. The spectrum of PKS 1510-089. MAGIC observations (open squares: observed; filled circles: EBL corrected using the model of Ref. 7) are shown together with the Fermi-LAT spectrum (open circles), obtained combining all events of time intervals coincident with the MAGIC observation window. The thick lines show the log parabola fit to the data and its extrapolation, where the thin lines indicate the statistical uncertainty of the fit. The dashed lines show the extrapolation with the EBL correction.

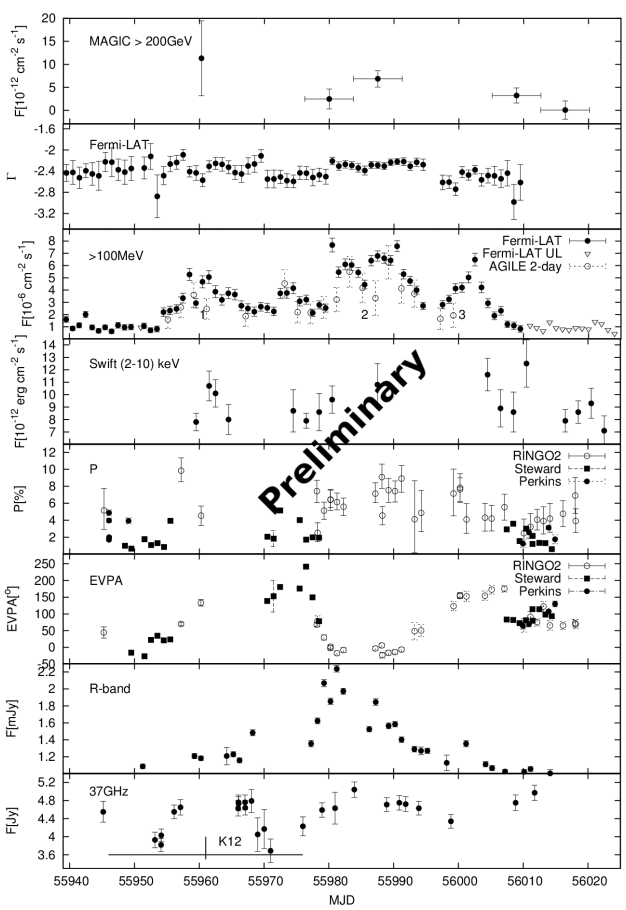

Fig. 4. MWL lightcurves of PKS 1510-089 in Spring 2012. The numbers in the Fermi-LAT lightcurve correspond to three distinct $\gamma$-ray flares, which show different MWL behavior. The symbol marked "K12" in the bottom panel shows the zero separation epoch of a knot from the VLBA core. 
$2.8 \pm 0.6$, Fig. 3 ) and the source did not show any variability neither at daily nor at weekly time-scales (upper panel of Fig. 4). These results are in agreement with those found by the H.E.S.S. collaboration during a previous observation campaign. ${ }^{2}$ The MWL lightcurves are presented in Fig. 4. During the MAGIC observation period, there were three distinct flares in $\mathrm{HE} \gamma$ rays. The latter were also detected by the AGILE telescope. All three flares are characterized by different behavior at lower energies. Simultaneously to the first HE flare, there was a rotation of $>180^{\circ}$ of the optical polarization angle, the ejection of a new radio knot and a radio flare. The second HE flare was accompanied by an optical flare, a rotation of $>180^{\circ}$ of the optical polarization angle but in the opposite direction with respect to the previous one, and a radio flare. The third HE flare had only a counterpart in the optical polarization angle: there was a rotation of $>180^{\circ}$ in the direction of the first rotation. During the whole MAGIC period, there was only modest activity in the X-ray band. HE and VHE spectra connected smoothly (Fig. 3), suggesting a negligible amount of internal absorption. We estimate that the emission region is located at the radio core, and we interpret the observed behavior using the scenario proposed by Ref. 9. A knot, propagating along the jet passes the VLBA core (flare 1 ). Flare 2 is caused by a sudden energization of the electrons in the knot and flare 3 by a increase in the local seed photon field. Regarding the optical polarization data, we assume the reason to be turbulence. The MWL SED (Fig. 5) has been modeled using two different leptonic scenarios: ${ }^{8}$ external low energy photons from the IR torus (left panel), and from a slow sheath of the jet (right panel).
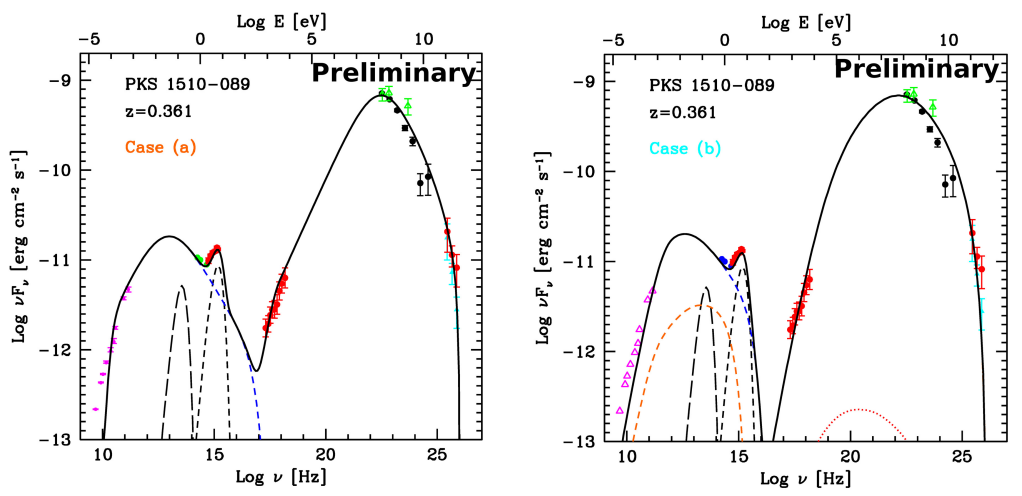

Fig. 5. The SED of PKS 1510-089 in Spring 2012 including F-GAMMA and Metsähovi (magenta), GASP-WEBT (green filled circles), Swift-UVOT and XRT (red filled circles), Fermi-LAT (black filled circles), AGILE (green, open triangles) and MAGIC (red, EBL corrected) data. The solid black line represents the modeling of the broad band emission, assuming the low energy peak to be originate from synchrotron emission (blue dashed line), while the high energy bump is dominated by the external Compton mechanism. In case A, seed photons are the IR torus (long dashed line) photons, while in case B, are coming from a slow sheath of the jet (light-blue dashed line). The short dashed line indicates the thermal component from the accretion disk. 


\section{Conclusions}

We have presented MAGIC results and MWL observations of two FSRQs, 3C 279 and PKS 1510-089. For 3C 279, we have derived the most stringent upper limits on VHE $\gamma$-ray emission. We have interpreted the observed behavior with a two-zone leptonic model for the SED, whereas the optical polarization signature is explained via a bent trajectory with relativistic aberration. Previous VHE detections of this source happened during high optical and/or X-ray states, and the MWL properties observed in June 2011 were similar to those previously reported, with the difference that in the present case the VHE observations did not result in a detection. PKS 1510-089 showed no VHE variability, contrary to the other two FSRQs known to show intra-night variability. The observed behavior could be explained with an emission region located at the radio core, and the variability was associated with an emission feature moving across different zones whose turbulence could be the source of the observed signatures in optical polarization. The analysis of the MWL behavior of these two sources allowed us to put constraints on the location of the emission regions and to propose possible coherent scenarios (full details in Ref. 10, 11). However the origin of the VHE $\gamma$-ray flares in these two sources remains an open question.

\section{Acknowledgments}

We would like to thank the Instituto de Astrofísica de Canarias for the excellent working conditions at the Observatorio del Roque de los Muchachos in La Palma. The support of the German BMBF and MPG, the Italian INFN, the Swiss National Fund SNF, and the Spanish MICINN is gratefully acknowledged. This work was also supported by the CPAN CSD2007-00042 and MultiDark CSD2009-00064 projects of the Spanish Consolider-Ingenio 2010 programme, by grant DO02-353 of the Bulgarian NSF, by grant 127740 of the Academy of Finland, by the DFG Cluster of Excellence "Origin and Structure of the Universe", by the DFG Collaborative Research Centers SFB823/C4 and SFB876/C3, and by the Polish MNiSzW grant 745/N-HESS-MAGIC/2010/0.

The Fermi-LAT Collaboration acknowledges support from a number of agencies and institutes for both development and the operation of the LAT as well as scientific data analysis. These include NASA and DOE in the United States, CEA/Irfu and IN2P3/CNRS in France, ASI and INFN in Italy, MEXT, KEK, and JAXA in Japan, and the K. A. Wallenberg Foundation, the Swedish Research Council and the National Space Board in Sweden. Additional support from INAF in Italy and CNES in France for science analysis during the operations phase is also gratefully acknowledged.

The AGILE Mission is funded by ASI with scientic and programmatic participation by INAF and INFN. 
The OVRO 40-m monitoring program is supported in part by NASA grants NNX08AW31G and NNX11A043G, and NSF grants AST-0808050 and AST1109911.

\section{References}

1. J. Albert et al., Science 320, 5884, 1752 (2008)

2. A. Abramowski et al., A\&S 5554, A107 (2013)

3. J. Cortina (for the MAGIC Collaboration), ATel \#3975 (2012)

4. J. Aleksić et al., ApJ 730, L8 (2011a)

5. J. Aleksić et al., Astropart. Phys. 35, 435 (2012)

6. J. Aleksić et al., A\&SA 530, A4 (2011b)

7. A. Dominguez et al., MNRAS 410, 2556 (2011)

8. L. Maraschi and F. Tavecchio, ApJ 593, 667 (2003)

9. A. P. Marscher et al., ApJL 710, L126 (2010)

10. J. Aleksić et al., submitted to A\&A, arXiv:1311.2833 [astro-ph.HE] (2013)

11. J. Aleksić et al., in prep. 